\title{
Lower recurrence rate with heavyweight mesh compared to lightweight mesh in laparoscopic totally extra-peritoneal (TEP) repair of groin hernia: a nationwide population-based register study
}

\author{
M. Melkemichel ${ }^{1,2}$ D S. Bringman ${ }^{1,2} \cdot$ B. Widhe ${ }^{1,2}$
}

Received: 28 April 2018 / Accepted: 18 August 2018 / Published online: 24 August 2018

(c) The Author(s) 2018

\begin{abstract}
Purpose Lightweight meshes (LWM) have shown benefits compared to heavyweight meshes (HWM) in terms of less postoperative pain and stiffness in open inguinal hernia repair. It appears to have similar advantages also in TEP, but concerns exist if it may be associated with higher recurrence rates. The aim of the study was to compare reoperation rate for recurrence of LWM to HWM in laparoscopic totally extra-peritoneal (TEP) repair.

Methods All groin hernias operated on with TEP between 1 January 2005 and 31 December 2013 at surgical units participating in The Swedish Hernia Register were eligible. Data included clinically important hernia variables. Primary endpoint was reoperation for recurrence. Median follow-up time was 6.1 years (0-11.5) with minimum 2.5 years postoperatively.

Results In total, 13,839 repairs were included for statistical analysis and 491 were re-operated for recurrence. Multivariate analysis demonstrated significantly increased risk of reoperation for recurrence in LWM 4.0\% (HR 1.56, $P<0.001$ ) compared to HWM 3.2\%. This was most evident in direct hernias (HR 1.75, $P<0.001)$ and in hernia repairs with a defect $>3 \mathrm{~cm}$ (HR $1.54, P<0.021)$. The risk of recurrence with use of LWM in indirect hernias and in hernia repairs with a defect $<1.5 \mathrm{~cm}$ was more comparable to HWM.

Conclusions Lightweight meshes were associated with an increased risk of reoperation for recurrence compared to HWM. While direct hernias and larger hernia defects may benefit from HWM to avoid increased recurrence rates, LWM is recommended to be used in indirect and smaller hernia defects in TEP repair.
\end{abstract}

Keywords Heavyweight mesh · Lightweight mesh - TEP · Groin hernia repair

\section{Introduction}

Prosthetic reinforcement has become the standard treatment of groin hernias compared to suture repair, since it significantly reduces the risk of recurrence and chronic pain [1]. However, the first generations of meshes, commonly polypropylene and polyester, were associated with side effects

Preliminary data were presented at the European Hernia Society Meeting 2017 in Vienna, Austria, and at the Swedish Surgical Society Meeting 2017 in Jönköping, Sweden.

M. Melkemichel

maria.melkemichel@ki.se

1 Department of Clinical Sciences, Karolinska Institutet, Danderyd Hospital, Stockholm, Sweden

2 Department of Surgery, Södertälje Hospital, 15286 Södertälje, Sweden such as pain and reduced abdominal wall compliance [2]. This has resulted in less foreign material in the development of modern meshes. The main properties of the mesh are found to be the type of filament, tensile strength, and porosity [3]. These can affect the weight of the mesh and its biocompability to the abdominal wall when it comes to flexibility and discomfort [3]. When decreasing the amount of polypropylene and increasing the pore size, less foreign body reaction is produced [4]. The so-called lightweight meshes (LWM) can, therefore, provide adequate strength for hernia repair with less associated side effects [5]. The previous studies have shown benefits of LWM compared to heavyweight meshes (HWM) in terms of improved aspects of pain, discomfort, and early return to normal activity after surgery in open anterior mesh groin hernia repair (Lichtenstein) $[6,7]$. LWM appears to have similar advantages in laparoscopic totally extra-peritoneal repair (TEP), as well 
[8]. However, concerns exist if it may have an increased risk of recurrence, especially for larger hernia defects $[9,10]$.

The aim of the study was to compare the reoperation for recurrence rate in a large number of hernias with long-term follow-up following the use of LWM versus standard HWM in TEP. The hypothesis was that LWM does not increase reoperation for recurrence rate compared to HWM.

\section{Methods}

The current study is a nationwide population-based register cohort that included all patients, 15 years or older, operated on with laparoscopic totally extra-peritoneal repair (TEP) between 1 January 2005 and 31 December 2013 at surgical units participating in The Swedish Hernia Register (SHR). The follow-up time was until 30 June 2016. Approval of the study protocol was obtained 22 June 2016 by the Regional Ethics Review Board in Stockholm, Sweden (DNR 2016/1144-31/2). Only aggregated data were collected without any accesses to sensitive information.

\section{The Swedish Hernia Register}

SHR was established in 1992 in eight Swedish hospitals and has gradually expanded. Currently, nearly $100 \%$ of all groin hernia repairs from over 90 units are registered. A total number of more than 350,000 operations have been registered [11, 12]. The SHR is a prospective voluntary register in which the patients are recorded with a personal identity number (PIN), unique for each citizen in Sweden. This allows linking with the previous hernia repairs registered in the SHR. Data include patient characteristics, type of hernia, size of the hernia defect, type of repair, type of mesh, operation time, complications within 30 days, and reoperations. The SHR is validated and $10 \%$ of the aligned units are checked independently every year. It is also connected to The Migration and The Cause of Death registers in Sweden.

\section{Material}

Clinically important variables that could affect the outcome were analyzed from the SHR, such as age, gender, ASA classification, hernia location in the groin, primary/recurrent hernia, unilateral/bilateral hernia, size of the hernia defect, type of mesh, mesh fixation, and reoperation for recurrence. The time from the TEP hernia repair and the reoperation for recurrence was calculated in days. Primary endpoint was reoperation for recurrence. It was defined as a new hernia repair in the same groin as a previous TEP repair was performed with a registration in the SHR. The hernia repair with TEP was either a primary or recurrent hernia, unilateral or bilateral. We included only one reoperation for recurrence for each groin in each patient after TEP.

\section{Type of meshes}

Heavyweight meshes (HWM) were defined as a polypropylene (PP) mesh with a weight $>50 \mathrm{~g} / \mathrm{m}^{2}$. Lightweight meshes (LWM) consisted of; a PP lightweight mesh with a weight $<50 \mathrm{~g} / \mathrm{m}^{2}$ or a composite PP lightweight mesh with monofilament made of absorbable poliglecaprone- 25 with a weight $<30 \mathrm{~g} / \mathrm{m}^{2}$ after absorption. Preformed meshes consisted of both heavyweight and lightweight meshes without a possible separation from the SHR, and were, therefore, excluded. In addition, meshes with less than 500 repairs were excluded and are reported in Fig. 1.

\section{Statistical analysis}

Descriptive statistics were used to describe baseline characteristics. Median values with range were given. Reoperation rates were illustrated using Kaplan-Meier hazards plots to assess the analysis of time from the TEP repair to reoperation for recurrence. Relative risks were estimated using Cox's proportional hazards model. First, univariate analysis of risk co-variables was calculated. All variables from the univariate model were then selected for the multivariate analysis (stratified for sex and type of hernia) to assess independent risk factors. In addition, a subgroup analysis was made for the meshes against risk co-variables. Confidence intervals (CI) at $95 \%$ were calculated. Statistical significance was considered at a $P$ value $<0.05$. All analyses were performed using R Core Team 2017 with survival and ggplot2 packages (Version 3.4.1. R: A language and environment for statistical computing. R Foundation for Statistical Computing, Vienna, Austria. URL https://www.R-project.org/). Cox. zph was calculated to exclude time-depended co-variates.

\section{Results}

A total of 17,348 groin hernia repairs were operated on with TEP during the 9-year inclusion period. After exclusion, 13,839 repairs remained for statistical analysis. A total number of 491 hernias were re-operated for recurrence. The overall follow-up time in the study material was 11.5 years with a minimum of 2.5 years postoperatively. The median follow-up time for all hernias was 6.1 years. $61.5 \%$ of the reoperations were performed within 2 years after the TEP operation. The overall cumulative reoperation rate in the material was 3.5\% Kaplan-Meier analysis of time from the TEP repair to reoperation for recurrence of the meshes which are illustrated in Fig. 2a. Hernia characteristics in the total and reoperation group are given in Table 1. 
Fig. 1 Flowchart of included hernias. $P P$ polypropylene, $H W M$ heavyweight meshes, $L W M$ lightweight meshes, ePTFE expanded polytetrafluoroethylene

\section{Eligible hernias}

Groin hernia TEP repairs registered in SHR 1 January 2005 - 31 December 2013 $(\mathrm{n}=17349)$

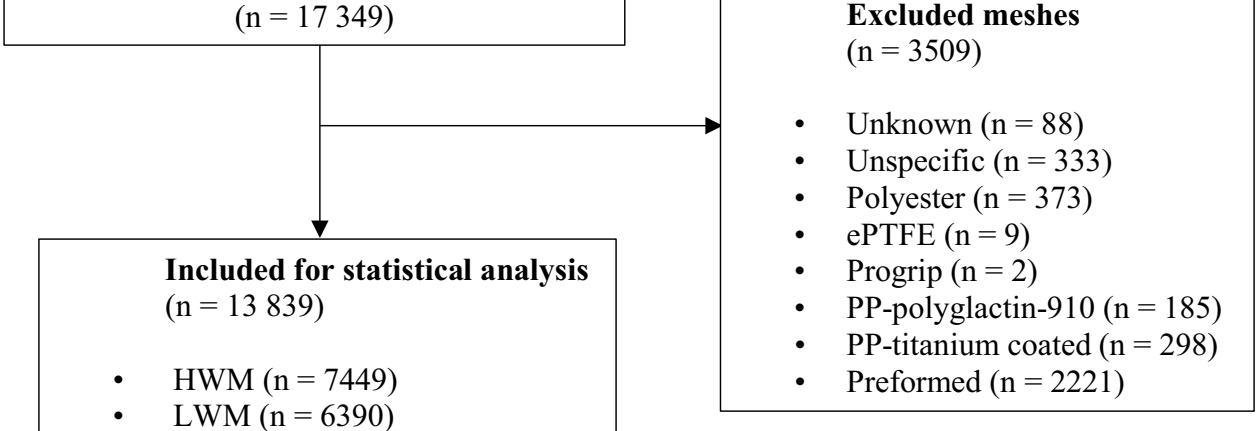

\section{Univariate and multivariate analyses}

HWM was used in 7449 of the TEP repairs and 235 of them were re-operated for recurrence. The median follow-up time for the HWM group was 88.2 months ( $0-11.5$ years). LWM was used in 6390 of the TEP repairs and 256 of them were re-operated for recurrence. The median follow-up time for the LWM group was 56.8 months ( $0-11.5$ years). Multivariate analysis demonstrated a significant increased risk of reoperation for recurrence in LWM 4.0\% (HR 1.56, 95\% CI 1.29-1.88; $P<0.001$ ) compared to HWM $3.2 \%$ (Fig. 2a; Table 2).

Both univariate and multivariate statistical analyses demonstrated a significant higher risk of reoperation for recurrence if age above median (HR 1.33, 95\% CI 1.10-1.60; $P=0.003$ ) (Fig. 2b; Table 2). 12.2\% were women in the total group and $5.9 \%$ in the reoperation group, demonstrating a significant decreased risk of reoperation for recurrence in the univariate analysis with a HR 0.39 (95\% CI 0.26-0.59; $P<0.001$ ) in female hernia repairs. $6 \%$ of the female hernias defect exceeded $3 \mathrm{~cm}$ compared to $22 \%$ in the male hernias. Femoral defects were more present in female hernias compared to male hernias, 27 versus $3 \%$.

Hernia repairs with a defect $>3 \mathrm{~cm}$ (HR $1.40,95 \%$ CI $1.06-1.86 ; P=0.019)$ was a risk factor of reoperation for recurrence in the univariate analysis, but not in the multivariate model.

There were no significant statistical differences of reoperation for recurrence rates after TEP in unilateral versus bilateral hernia $(P=0.985)$, type of defect (direct versus indirect versus femoral versus combined), if the index operation was a primary or recurrent hernia $(P=0.680)$ or if mesh fixation or no fixation was used $(P=0.960)$ (Table 2).

\section{Subgroup analysis}

\section{Age}

The multivariate analysis showed that recurrences were more frequent in the older half of the study population (patients $\geq 59$ years), than in the younger half (patients $<59$ years) (Fig. 2b; Table 2). However, the disparity in reoperations rate for recurrence between the meshes was more evident in the younger half (Table 3).

\section{Type of defect}

In direct male hernias, the reoperation for recurrence rate was significantly higher in LWM than in HWM (Fig. 2c; Table 3). However, the dissimilarity between LWM and HWM was most apparent in the younger half ( $<59$ years) (Fig. 2d; Table 3). In indirect male hernia repairs, the reoperation for recurrence rate was more similar between the meshes (Table 3 ). 


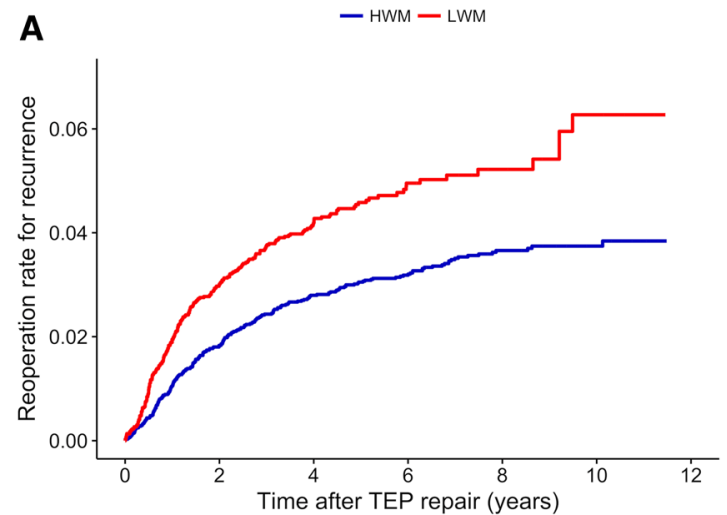

Number at risk

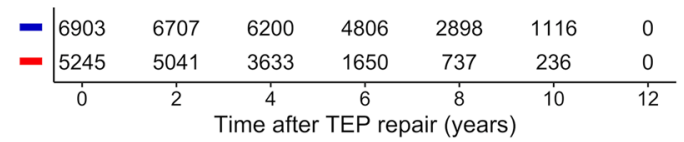

C

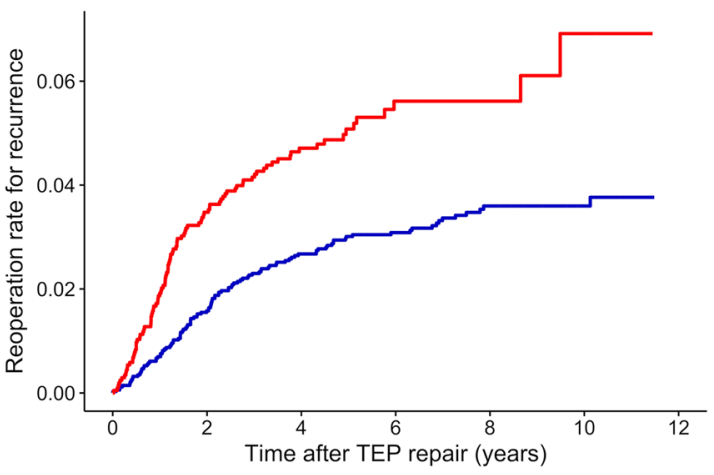

Number at risk
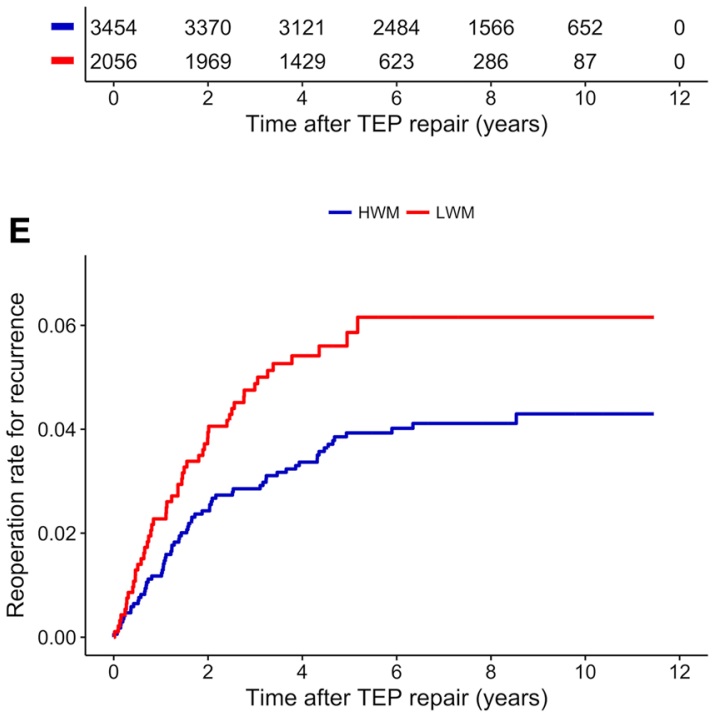

Number at risk

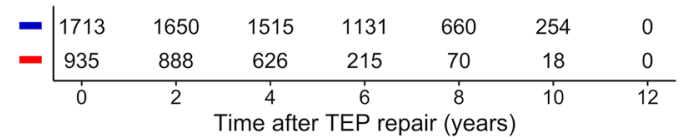

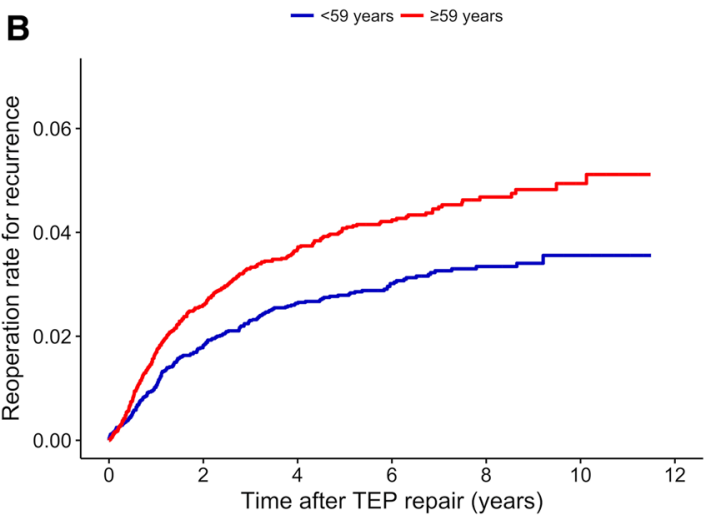

Number at risk

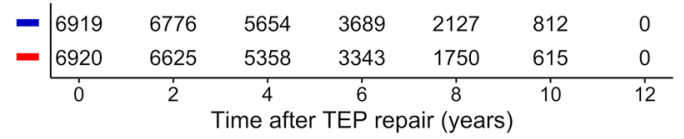

D $\quad-\mathrm{HWM}^{-\mathrm{LWM}}$

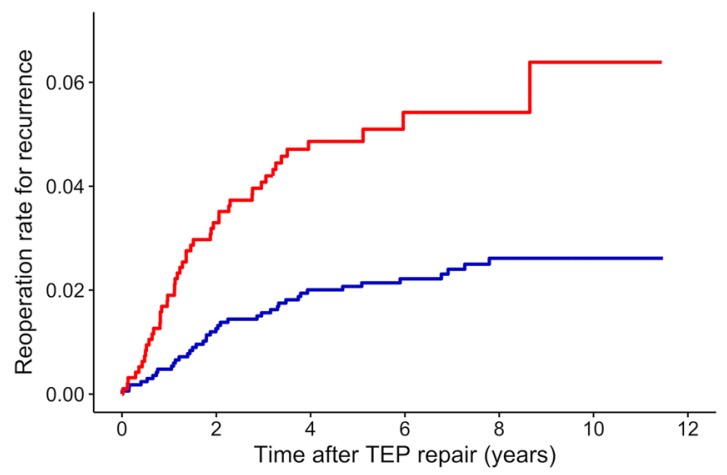

Number at risk

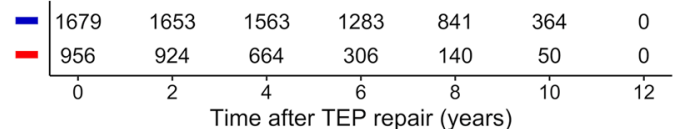


4Fig. 2 Kaplan-Meier hazard plot illustrating time from the TEP repair to reoperation rate for recurrence in a male hernia repairs, $\mathbf{b}$ age (divided by median), $\mathbf{c}$ direct hernias in male patients, $\mathbf{d}$ direct hernias in male patients $<59$ years, and e male hernias $>3 \mathrm{~cm}$. a $P<0.001$, b $P<0.001$, c $P<0.001$, d $P<0.001$, and e $P=0.020$ (logrank test)

\section{Size of defect}

Hernias with a defect exceeding $3 \mathrm{~cm}$ demonstrated a higher risk of reoperation for recurrence in LWM compared to HWM (Table 3; Fig. 2e). Hernias with a defect $<1.5 \mathrm{~cm}$ resulted in no difference between the meshes (Table 3 ).

\section{Discussion}

This study is, to our knowledge, the largest cohort that compares the risk of reoperation for recurrence in lightweight and heavyweight meshes in TEP repair with a long follow-up.

Recurrence is an undesirable complication after groin hernia repair and our hypothesis was that LWM was comparable to HWM in respect of recurrence rates. This was not entirely supported in this report with 13,839 hernias operated in Sweden, recruited from the Swedish Hernia Register. Instead, we detected an increased risk of recurrence after use of LWM compared to HWM both in the univariate and multivariate statistical analyses. The difference in recurrence rate between the mesh groups could be considered as small and overall low. The total reoperation rate for recurrence in our material was 3.5 per cent. This corresponds well with the findings published by the International Endo Hernia Society's (IEHS) guidelines reporting an incidence even lower at specialized centers [13, 14].

The previous studies have reported a higher recurrence rate after TEP repair with LWM compared to HWM, although not statistically significant [9]. However, Burgmans et al. reported in the recent randomized controlled trial with 2-year follow-up not only a significant higher recurrence rate in LWM, but also an increased rate of chronic pain compared to HWM [15]. The IEHS's recommendation is that meshes with large pores are more beneficial to use than meshes with more foreign body material [14]. The theory is that HWM is oversized with regards to the mechanical properties that can lead to more postoperative pain and stiffness [16, 17]. The findings are summarized from controlled-randomized studies, with short-to-medium-term results having different postoperative aspects as primary outcome $[8,18,19]$. However, these studies have been undersized in terms of number of patients and have had insufficient follow-up time to detect a difference in recurrence rate between HWM and LWM. Therefore, the strength of our study is the large number of unselected TEP hernia repairs conducted during a 9-year period. The data from SHR represent both low- and highvolume TEP surgeons from almost the whole nation, eliminating single surgeon and possible center bias, as opposed to TEP experts that normally are operating in randomized clinical trials. A randomized control trial is considered to be the golden standard to establish reliable clinical results. However, since recurrence rate is low with only a few percent presenting recurrences after 3 years of follow-up, a good RCT has limitations to implement the number of hernia repairs that requires demonstrating a significant difference between the meshes. Our study with 13,839 TEP hernia repairs over an 11.5-year period makes it possible to analyze risk factors of reoperation for recurrence with a long follow-up. However, the study has limitations. First, patientspecific information such as smoking, obesity, medications, or status of physical activity was absent, which may have had an impact on outcome. Second, the surgeon's technical skills in performing TEP repairs could also affect the outcome and could not be evaluated in this study. However, in all centers participating in the current study, the use of LWM resulted in higher reoperation rates for recurrence compared to HWM. Nearly, all repairs were performed by consultant surgeons and not by residents. Third, another limitation with the study is the lack of information of the mesh size. The register does not provide that data and the previous reports have shown that appropriate size may be more important to avoid recurrence than the surgical technique and type of mesh [14]. Finally, a circumscription in this study was the use of reoperation rate for recurrence as a surrogate measure for the true recurrence rate. The previous reports presents data, showing that reoperation rate could be underestimated with $40 \%$ [20].

Heavyweight meshes made of polypropylene has been used for a long period of time and the reason for a change to LWM is to avoid side effects of pain, stiffness, and foreign body sensation that could be associated with HWM [21]. A mesh should have adequate strength, be of an appropriate size, and attain good tissue incorporation by initiation of fibrosis to prevent recurrence [5]. The explanation for the increase of the recurrence rate with LWM compared to HWM in our study could be partly explained by the higher intrinsic weakness and the decreased formation of fibrosis that has been showed in animal experiments [22]. The mass of fibrosis that is formed depends on the intensity of inflammatory response, whereas the two most important factors for this are the pore size and the amount of foreign material [22]. The inflammatory response continues 3 months postoperatively and the following fibrotic changes can lead to that pain may occur later [23]. Therefore, it is theoretically considered that increased fibrotic reaction from the use of HWM with more material would be accompanied by a higher frequency of chronic pain [16]. The current literature 
Table 1 Baseline hernia characteristics in each mesh group

\begin{tabular}{|c|c|c|c|c|c|c|}
\hline \multirow[t]{2}{*}{ Type of mesh } & \multicolumn{3}{|l|}{ Total group } & \multicolumn{3}{|l|}{ Reoperation group } \\
\hline & HWM & LWM & Total & HWM & LWM & Total \\
\hline No. of hernias & $7449(53.8)$ & $6390(46.2)$ & 13,839 & $235(47.9)$ & $256(52.1)$ & 491 \\
\hline Age, years ${ }^{\mathrm{a}}$ (range) & $59.4(15.4-93.8)$ & $58.8(15.0-94.5)$ & $59.2(15.0-94.5)$ & $62.3(19.4-86.9)$ & $61.0(15.3-89.2)$ & $61.3(15.3-89.2)$ \\
\hline \multicolumn{7}{|l|}{ ASA class } \\
\hline I-II & $7062(94.8)$ & $6006(94.0)$ & $13,068(94.4)$ & $224(95.3)$ & $235(91.8)$ & $459(93.5)$ \\
\hline III-IV & $365(4.9)$ & $382(6.0)$ & $747(5.4)$ & $11(4.7)$ & $21(8.2)$ & $32(6.5)$ \\
\hline NA & $22(0.3)$ & $2(0.0)$ & $24(0.2)$ & 0 & 0 & 0 \\
\hline \multicolumn{7}{|l|}{ Sex } \\
\hline Male & 6903 (92.7) & $5245(82.1)$ & $12,148(87.8)$ & 232 (98.7) & $235(91.8)$ & $467(95.1)$ \\
\hline Female & $546(7.3)$ & $1145(18.0)$ & $1691(12.2)$ & $3(1.3)$ & $21(8.2)$ & $24(5.1)$ \\
\hline \multicolumn{7}{|l|}{ Type of hernia } \\
\hline Bilateral & $4877(65.5)$ & $3488(54.6)$ & $8365(60.4)$ & $161(68.5)$ & $140(54.7)$ & $301(61.3)$ \\
\hline Unilateral & $2572(34.5)$ & $2902(45.4)$ & 5474 (39.6) & $74(31.5)$ & $116(45.3)$ & $190(38.7)$ \\
\hline \multicolumn{7}{|l|}{ Recurrent hernia } \\
\hline No & $5867(78.8)$ & $5227(81.8)$ & $11,094(80.2)$ & $184(78.3)$ & 204 (79.7) & $388(79.0)$ \\
\hline Yes & $1582(21.2)$ & $1163(18.2)$ & 2745 (19.7) & $51(21.7)$ & $52(20.3)$ & $103(21.0)$ \\
\hline \multicolumn{7}{|l|}{ Type of defect } \\
\hline Indirect & $3047(41.0)$ & $3218(50.4)$ & $6265(45.3)$ & $96(40.9)$ & $115(44.9)$ & $211(43.0)$ \\
\hline Direct & $3532(47.4)$ & $3314(51.9)$ & $6846(49.5)$ & $113(48.1)$ & $105(41.0)$ & $218(44.4)$ \\
\hline Femoral & $199(2.7)$ & $319(5.0)$ & $518(3.7)$ & $4(1.7)$ & $10(3.9)$ & $14(2.9)$ \\
\hline Combined/others & $634(8.9)$ & $565(8.8)$ & $1199(8.7)$ & $22(9.3)$ & $23(9.0)$ & $45(9.2)$ \\
\hline NA & $37(0.5)$ & $110(1.7)$ & $147(1.1)$ & 0 & $3(1.2)$ & $3(0.6)$ \\
\hline \multicolumn{7}{|l|}{ Size of defect } \\
\hline$<1.5 \mathrm{~cm}$ & $1174(16.0)$ & $1520(23.8)$ & 2694 (19.5) & $33(14.0)$ & $49(19.1)$ & $82(16.7)$ \\
\hline $1.5-3 \mathrm{~cm}$ & $4126(55.3)$ & $3880(60.7)$ & $8006(57.9)$ & $123(52.3)$ & $155(60.5)$ & $278(56.6)$ \\
\hline$>3 \mathrm{~cm}$ & $1764(23.7)$ & $984(15.4)$ & $2748(19.9)$ & $67(28.5)$ & $52(20.3)$ & $119(24.2)$ \\
\hline NA & $385(5.2)$ & $6(0.0)$ & $391(2.8)$ & $12(5.1)$ & 0 & $12(2.4)$ \\
\hline \multicolumn{7}{|l|}{ Mesh fixation } \\
\hline Yes & $4236(56.9)$ & $4434(69.3)$ & $8670(62.6)$ & $152(64.7)$ & $169(66.0)$ & $321(65.4)$ \\
\hline No & $3213(43.1)$ & 1956 (30.6) & 5169 (37.4) & $83(35.3)$ & $87(34.0)$ & $170(34.6)$ \\
\hline
\end{tabular}

Values in parentheses are in percentage unless indicated otherwise

NA unknown/missing, HWM heavyweight meshes, LWM lightweight meshes, ASA class American Society of Anesthesiologists classification

${ }^{a}$ Age are given in median with range

regarding pain in the use of LWM or HWM shows no significant differences at 1-year follow-up after TEP [9, 24-26]. In contrast, Li et al. showed higher incidence of chronic groin pain after 6-month follow-up after use of HWM compared to LWM [27]. The authors were also concerned about the results showing increased recurrence for LWM, especially for larger defects. Despite the conflicting data concerning pain after use of LWM compared to HWM in TEP, according to recent guidelines, one should at least avoid fixation in TEP, as fixation itself may result in chronic pain without preventing recurrence [14, 28]. In our study, the non-fixated meshes did not reveal an increased risk of reoperation for recurrence compared to the fixated ones. The tendency was instead that, in the TEP repairs where the mesh was fixated, it resulted in an increased reoperation rate for recurrence. An explanation for this result may be that a higher proportion of the procedure with a suboptimal dissection for the mesh had mesh fixation.

Another interesting result in the current study was the detection of older patients having an increased risk of reoperation for recurrence. Whereas most studies have not revealed age as a significant risk factor for recurrence, in our study, age above median ( $\geq 59$ years) was significantly related to a higher risk of reoperation for recurrence. The reason why patients over 59 years had a higher recurrence rate is not yet clear. One possible explanation could be that older patients have a weaker connective tissue quality, thereby increasing the risk of recurrence. We also believe that the recurrence rate is probably underestimated particularly in older patients. It may possibly be due to not seeking 
Table 2 Univariate and multivariate Cox's proportional hazard ratio analysis of risk factors of reoperation for recurrence

\begin{tabular}{|c|c|c|c|c|c|c|}
\hline \multirow[t]{2}{*}{ Variable } & \multicolumn{2}{|c|}{ Reoperation rate } & \multicolumn{2}{|l|}{ Univariate analysis } & \multicolumn{2}{|c|}{ Multivariate analysis* } \\
\hline & $(n)$ & $(\%)$ & Hazard ratio & $P$ & Hazard ratio & $P$ \\
\hline \multicolumn{7}{|l|}{ Age, years } \\
\hline$<59$ & $207 / 6919$ & 3.0 & 1.00 (reference) & & 1.00 (reference) & \\
\hline$\geq 59$ & $284 / 6920$ & 4.1 & $1.41(1.18-1.69)$ & $<\mathbf{0 . 0 0 1}$ & $1.33(1.10-1.60)$ & 0.003 \\
\hline \multicolumn{7}{|l|}{ ASA class } \\
\hline I-II & $459 / 13,068$ & 3.5 & 1.00 (reference) & & 1.00 (reference) & \\
\hline III-IV & $32 / 747$ & 4.3 & $1.33(0.93-1.91)$ & 0.116 & $1.20(0.84-1.73)$ & 0.320 \\
\hline \multicolumn{7}{|l|}{ Sex } \\
\hline Male & $467 / 12,148$ & 3.8 & 1.00 (reference) & & & \\
\hline Female & $24 / 1691$ & 1.4 & $0.39(0.26-0.59)$ & $<0.001$ & & \\
\hline \multicolumn{7}{|l|}{ Type of mesh } \\
\hline HWM & $235 / 7449$ & 3.2 & 1.00 (reference) & & 1.00 (reference) & \\
\hline LWM & $256 / 6390$ & 4.0 & $1.44(1.21-1.72)$ & $<0.001$ & $1.56(1.29-1.88)$ & $<0.001$ \\
\hline \multicolumn{7}{|l|}{ Type of hernia } \\
\hline Unilateral & $190 / 5474$ & 3.5 & 1.00 (reference) & & & \\
\hline Bilateral & $301 / 8365$ & 3.6 & $1.00(0.84-1.20)$ & 0.985 & & \\
\hline \multicolumn{7}{|l|}{ Type of defect } \\
\hline Direct & $218 / 5710$ & 3.8 & 1.00 (reference) & & 1.00 (reference) & \\
\hline Indirect & $211 / 6265$ & 3.4 & $0.91(0.75-1.10)$ & 0.308 & $0.96(0.79-1.18)$ & 0.722 \\
\hline Femoral & $27 / 808$ & 3.3 & $0.92(0.62-1.37)$ & 0.682 & $1.36(0.89-2.08)$ & 0.158 \\
\hline Combined/others & $35 / 1056$ & 3.3 & $0.89(0.62-1.27)$ & 0.519 & $0.86(0.60-1.23)$ & 0.406 \\
\hline \multicolumn{7}{|l|}{ Size of defect } \\
\hline$<1.5 \mathrm{~cm}$ & $82 / 2694$ & 3.0 & 1.00 (reference) & & 1.00 (reference) & \\
\hline $1.5-3 \mathrm{~cm}$ & $278 / 8006$ & 3.5 & $1.12(0.88-1.43)$ & 0.367 & $1.01(0.79-1.31)$ & 0.911 \\
\hline$>3 \mathrm{~cm}$ & $119 / 2748$ & 4.3 & $1.40(1.06-1.86)$ & 0.019 & $1.27(0.94-1.71)$ & 0.124 \\
\hline \multicolumn{7}{|l|}{ Recurrent hernia } \\
\hline No & $387 / 11,090$ & 3.5 & 1.00 (reference) & & 1.00 (reference) & \\
\hline Yes & $104 / 2749$ & 3.8 & $1.07(0.86-1.33)$ & 0.544 & $0.95(0.75-1.21)$ & 0.680 \\
\hline \multicolumn{7}{|l|}{ Mesh fixation } \\
\hline No & $170 / 5169$ & 3.3 & 1.00 (reference) & & 1.00 (reference) & \\
\hline Yes & $321 / 8670$ & 3.7 & $1.12(0.93-1.35)$ & 0.221 & $1.01(0.83-1.22)$ & 0.960 \\
\hline
\end{tabular}

Values in parentheses are $95 \%$ confidence intervals. Age is divided by median

Significant $P$ values are in bold face

ASA class American Society of Anesthesiologists classification

*Stratified for sex and type of hernia in the multivariate analysis care for the recurrent hernia and surgeons also being reluctant to re-operate older patients. Although the reoperation rate for recurrence was significantly higher in older patients, the difference between HWM and LWM was most evident in younger patients (Fig. 2d).

There was also a difference in the outcome between the genders. The reoperation rate for recurrence was in female hernia repair $1.4 \%$, significantly lower than for male patients. We could identify two possible main explanations to the difference between the genders. First, the size of the defect that exceeded $3 \mathrm{~cm}$ was much lower in female hernias compared to in male hernias. Second, the high proportion of femoral defects in female patients may cause difficulty to reoperate with an anterior repair and thereby, perhaps, lower the reoperation rate for recurrence. A pre-peritoneal repair, open or laparoscopic, is the golden standard in female groin hernia repair and is supported by the low reoperation rate for recurrence in female patients in this study.

The defect of the hernia was not a significant factor related to reoperation for recurrence. In addition, neither recurrent hernias nor unilateral or bilateral repairs demonstrated any significant differences. However, in the subgroup analysis of all direct hernias in men, we demonstrated a significant difference between the meshes (Fig. 2c). In the younger half of the male patients ( $<59$ years) with direct hernias, this was more evident (Fig. 2d) compared to the older group of direct hernias ( $\geq 59$ years), where there was no difference between the 
Table 3 Subgroup Cox's proportional hazard ratio analysis of reoperation for recurrence

\begin{tabular}{|c|c|c|c|c|}
\hline \multirow[t]{2}{*}{ Subgroup } & \multicolumn{2}{|c|}{$\begin{array}{l}\text { Reoperation } \\
\text { rate }\end{array}$} & \multicolumn{2}{|l|}{ LWM } \\
\hline & $(n)$ & $(\%)$ & Hazard ratio & $P$ \\
\hline \multicolumn{5}{|l|}{ Age, years } \\
\hline$<59$ & $195 / 5971$ & 3.3 & $1.70(1.28-2.27)$ & $<\mathbf{0 . 0 0 1}$ \\
\hline$\geq 59$ & $272 / 6177$ & 4.4 & $1.40(1.10-1.78)$ & 0.006 \\
\hline \multicolumn{5}{|l|}{ Type of defect } \\
\hline Direct & $215 / 5510$ & 3.9 & $1.75(1.33-2.29)$ & $<0.001$ \\
\hline $\begin{array}{l}\text { Direct in patients }<59 \\
\text { years }\end{array}$ & $87 / 2635$ & 3.3 & $2.39(1.56-3.66)$ & $<0.001$ \\
\hline $\begin{array}{l}\text { Direct in patients } \geq 59 \\
\text { years }\end{array}$ & $128 / 2875$ & 4.5 & 1.39 (0.98-1.98) & 0.065 \\
\hline Indirect & $198 / 5310$ & 3.7 & $1.33(1.01-1.76)$ & 0.045 \\
\hline \multicolumn{5}{|l|}{ Size of defect } \\
\hline$<1.5 \mathrm{~cm}$ & $72 / 1981$ & 3.6 & $1.32(0.83-2.11)$ & 0.245 \\
\hline$>3 \mathrm{~cm}$ & $118 / 2648$ & 4.5 & $1.54(1.07-2.22)$ & 0.021 \\
\hline
\end{tabular}

Values in parentheses are 95\% confidence intervals. HWM, reference. Women were excluded in all subgroup analyses. Age is divided by median. Significant $P$ values are in bold face

meshes. Thus, younger patients with a direct hernia had pronounced higher risk of reoperation for recurrence after the use of LWM.

Hence, in indirect hernias, the differences between the meshes were more comparable. Moreover, the use of LWM was not associated with an increased risk of reoperation for recurrence compared to HWM in smaller hernia defects (Table 3).

Furthermore, similar to several previous studies, we found that hernia repairs with a defect exceeding $3 \mathrm{~cm}$ were associated with an increased risk of reoperation for recurrence in the univariate analysis. The intrinsic weakness of LWM and the decreased formation of fibrosis may be a part of the increased hernia recurrences after the use of LWM. The less material in LWM may not have sufficient strength to avoid bulging in larger defects.

In conclusion, this first long-term nationwide populationbased study, comparing the use of LWM to HWM in TEP groin hernia repair, showed that LWM was associated with an increased risk of reoperation for recurrence. This was most evident in direct hernias (particularly in younger male patients) and in larger hernia defects. These might benefit from HWM to avoid increased recurrence rates. However, the overall difference in recurrence rate between the mesh groups was small, and therefore, other aspects, such as chronic pain, need to be considered when choosing type of mesh. The use of LWM could possibly improve other outcomes and are, therefore, recommended to be used in indirect hernias and in smaller hernia defects in TEP repair, since the risk of recurrence was comparable to HWM.
Acknowledgements The authors are thankful for statistical advice from the Swedish Hernia Registers statistician Henrik Holmberg, Umeå University, Sweden.

Funding The study was supported by Södertälje Hospital's SLL's and Capio's research and development funds. The funding source had no involvement in approving, planning, conducting, and analyzing the data or writing the final manuscript of the study.

\section{Compliance with ethical standards}

Conflict of interest S. B. declares potential conflict of interest not directly related to the submitted work (he is a member of the advisory board of the International Hernia Mesh Registry). For the remaining authors, none declares conflict of interest.

Ethical approval Approval of the study protocol was obtained 22 June 2016 by the Regional Ethics Review Board in Stockholm, Sweden (DNR 2016/1144-31/2). Only aggregated data were collected without any accesses to sensitive information.

Human and animal rights All procedures performed in the study involving human participants were in accordance with the ethical standards of the institutional and/or national research committee and with the 1964 Helsinki declaration and its later amendments or comparable ethical standards. This article does not contain any studies with animals performed by any of the authors.

Informed consent For this type of study, formal consent is not required. However, oral informed consent is obtained from all the participants for registration in the SHR.

Open Access This article is distributed under the terms of the Creative Commons Attribution-NonCommercial 4.0 International License (http://creativecommons.org/licenses/by-nc/4.0/), which permits any noncommercial use, distribution, and reproduction in any medium, provided you give appropriate credit to the original author(s) and the source, provide a link to the Creative Commons license, and indicate if changes were made.

\section{References}

1. (2000) Mesh compared with non-mesh methods of open groin hernia repair: systematic review of randomized controlled trials. Br J Surg 87:854-859. https://doi.org/10.104 6/j.1365-2168.2000.01539.x

2. Klinge U, Park J-KK, Klosterhalfen B (2013) The ideal mesh? Pathobiology 80:169-175. https://doi.org/10.1159/000348446

3. Brown CN, Finch JG (2010) Which mesh for hernia repair? Ann R Coll Surg Engl 92:272-278

4. Klinge U, Klosterhalfen B, Müller M, Schumpelick V (1999) Foreign body reaction to meshes used for the repair of abdominal wall hernias. Eur J Surg 165:665-673. https://doi.org/10.1080/11024 159950189726

5. Bringman S, Conze J, Cuccurullo D et al (2010) Hernia repair: the search for ideal meshes. Hernia 14:81-87. https://doi.org/10.1007/ s10029-009-0587-x

6. Bringman S, Wollert S, Osterberg J et al (2006) Three-year results of a randomized clinical trial of lightweight or standard polypropylene mesh in Lichtenstein repair of primary inguinal hernia. $\mathrm{Br}$ J Surg 93:1056-1059. https://doi.org/10.1002/bjs.5403 
7. Uzzaman MM, Ratnasingham K, Ashraf N (2012) Meta-analysis of randomized controlled trials comparing lightweight and heavyweight mesh for Lichtenstein inguinal hernia repair. Hernia 16:505-518. https://doi.org/10.1007/s10029-012-0901-x

8. Bringman S, Wollert S, Osterberg J, Heikkinen T (2005) Early results of a randomized multicenter trial comparing Prolene and VyproII mesh in bilateral endoscopic extraperitoneal hernioplasty (TEP). Surg Endosc 19:536-540. https://doi.org/10.1007/s0046 4-004-9100-x

9. Chowbey PK, Garg N, Sharma A et al (2010) Prospective randomized clinical trial comparing lightweight mesh and heavyweight polypropylene mesh in endoscopic totally extraperitoneal groin hernia repair. Surg Endosc 24:3073-3079. https://doi. org/10.1007/s00464-010-1092-0

10. Akolekar D, Kumar S, Khan LR et al (2008) Comparison of recurrence with lightweight composite polypropylene mesh and heavyweight mesh in laparoscopic totally extraperitoneal inguinal hernia repair: an audit of 1,232 repairs. Hernia 12:39-43. https:// doi.org/10.1007/s10029-007-0275-7

11. SHR (Swedish Hernia Register) website. http://www.svensktbra ckregister.se/ Accessed Aug 2018

12. Nilsson E, Haapaniemi S (1998) Hernia registers and specialization. Surg Clin N Am 78:1141-1155. https://doi.org/10.1016/ S0039-6109(05)70375-3 ix.

13. Bittner R, Arregui ME, Bisgaard T et al (2011) Guidelines for laparoscopic (TAPP) and endoscopic (TEP) treatment of inguinal hernia [International Endohernia Society (IEHS)]. Surg Endosc 25:2773-2843. https://doi.org/10.1007/s00464-011-1799-6

14. Bittner R, Montgomery MA, Arregui E et al (2015) Update of guidelines on laparoscopic (TAPP) and endoscopic (TEP) treatment of inguinal hernia (International Endohernia Society). Surg Endosc 29:289-321. https://doi.org/10.1007/s00464-014-3917-8

15. Burgmans JP, Voorbrood CE, Simmermacher RK et al (2016) Long-term results of a randomized double-blinded prospective trial of a lightweight (Ultrapro) versus a heavyweight mesh (Prolene) in laparoscopic total extraperitoneal inguinal hernia repair (TULP-trial). Ann Surg 263:862-866. https://doi. org/10.1097/SLA.0000000000001579

16. Schmidbauer S, Ladurner R, Hallfeldt KK, Mussack T (2005) Heavy-weight versus low-weight polypropylene meshes for open sublay mesh repair of incisional hernia. Eur J Med Res 10:247-253

17. Welty G, Klinge U, Klosterhalfen B et al (2001) Functional impairment and complaints following incisional hernia repair with different polypropylene meshes. Hernia 5:142-147

18. Heikkinen $T$, Wollert $S$, Osterberg $J$ et al (2006) Early results of a randomised trial comparing Prolene and VyproII-mesh in endoscopic extraperitoneal inguinal hernia repair (TEP) of recurrent unilateral hernias. Hernia 10:34-40. https://doi.org/10.1007/ s10029-005-0026-6

19. Agarwal BB, Agarwal KA, Mahajan KC (2009) Prospective double-blind randomized controlled study comparing heavy- and lightweight polypropylene mesh in totally extraperitoneal repair of inguinal hernia: early results. Surg Endosc 23:242-247. https ://doi.org/10.1007/s00464-008-0188-2

20. Kald A, Nilsson E, Anderberg B et al (1998) Reoperation as surrogate endpoint in hernia surgery. A three year follow-up of 1565 herniorrhaphies. Eur J Surg 164:45-50. https://doi. org/10.1080/110241598750004940

21. Khan LR, Liong S, Beaux AC de et al (2010) Lightweight mesh improves functional outcome in laparoscopic totally extraperitoneal inguinal hernia repair. Hernia 14:39-45. https://doi. org/10.1007/s10029-009-0558-2

22. Junge K, Klinge U, Rosch R et al (2002) Functional and morphologic properties of a modified mesh for inguinal hernia repair. World J Surg 26:1472-1480. https://doi.org/10.1007/s0026 8-002-6444-Z

23. Donati M, Brancato G, Grosso G et al (2016) Immunological reaction and oxidative stress after light or heavy polypropylene mesh implantation in inguinal hernioplasty: a CONSORT-prospective, randomized, clinical trial. Medicine (Baltimore) 95:e3791. https ://doi.org/10.1097/MD.0000000000003791

24. Khan LR, Kumar S, Nixon SJ (2006) Early results for new lightweight mesh in laparoscopic totally extra-peritoneal inguinal hernia repair. Hernia 10:303-308. https://doi.org/10.1007/s1002 9-006-0093-3

25. Lauscher JC, Yafaei K, Buhr HJ, Ritz JPP (2008) Total extraperitoneal hernioplasty: does the long-term clinical course depend on the type of mesh? J Laparoendosc Adv Surg Tech A 18:803-808. https://doi.org/10.1089/lap.2008.0036

26. Currie A, Andrew H, Tonsi A et al (2012) Lightweight versus heavyweight mesh in laparoscopic inguinal hernia repair: a metaanalysis. Surg Endosc 26:2126-2133. https://doi.org/10.1007/ s00464-012-2179-6

27. Li J, Ji Z, Cheng T (2012) Lightweight versus heavyweight in inguinal hernia repair: a meta-analysis. Hernia 16:529-539. https ://doi.org/10.1007/s10029-012-0928-z

28. Taylor C, Layani L, Liew V et al (2008) Laparoscopic inguinal hernia repair without mesh fixation, early results of a large randomised clinical trial. Surg Endosc 22:757-762. https://doi. org/10.1007/s00464-007-9510-7 